\title{
EFFECTS OF ELECTROMAGNETIC INTERFERENCE CAUSED BY LIGHTING TECHNOLOGIES IN BB-PLC SYSTEMS
}

\author{
Keyla Rezende Cardoso ${ }^{1}$, Paulo Vinicius Alves Freitas ${ }^{2}$, Pedro Vladimir Gonzalez Castellanos ${ }^{3}$, \\ Joacir Oliveira Silva ${ }^{4}$ and Marcio Zamboti Fortes ${ }^{5}$
}

1, 2, 3, 4, 5 Federal University Fluminense - UFF. Rio de Janeiro - Rio de Janeiro, Brazil.

Email: keylarezendec@gmail.com, pvafreita@gmail.com, pcastellanos@id.uff.br, joacir.uff@gmail.com, mzamboti@id.uff.br

Received: Feb 29th, 2020

Accepted: Apr 13 ${ }^{\text {th }}, 2020$

Published: April 30 $30^{\text {th }}, 2020$

Copyright (C2016 by authors and Galileo Institute of Technology and Education of the Amazon (ITEGAM).

This work is licensed under the Creative Commons Attribution International License (CC BY 4.0).

https://creativecommons.org/licen ses/by/4.0/

\begin{abstract}
Powerline communication (PLC) is a promising technology that allows data transmission through electric power distribution networks, with the advantage of using an existing infrastructure, reducing costs in maintenance and installation of telecommunication systems. However, once power systems are not designed specifically for data transmission, it may exhibit limiting electromagnetic interference characteristics for desirable system performance. This paper aims to analyze data rate performance of a Broadband PLC system when operating under conditions of conducted and radiated electromagnetic interference caused by lighting devices. The analyzed data transmission system presented good performance when subject to radiated electromagnetic interference, but was shown to be sensitive to conducted disturbances caused by lighting devices.
\end{abstract}

Keywords: Powerline Communication, Electromagnetic Interference, Data Rate, LED Lamps, Compact Fluorescent Lamps.

\section{INTRODUCTION}

The use of telecommunication systems has been growing rapidly in recent decades [1] and, responsively, new technologies and applications arise in the market aiming to meet demands of the sector's evolution.

The telecommunication systems became an important element in power distribution systems operation using Smart Grids. This type of network is a distribution architecture that allows bidirectional communication, together with the energy flow, among system stakeholders (end user and network, for example) [2]. Such network architecture has been gaining space in the electric sector in recent years, mainly with the technological advances coming from the telecommunication systems.

One of the telecommunications technologies applicable to the systems automation and communication is the data transmission through electric power supply networks, the Powerline Communication (PLC) technology, that brings as main advantage the reduction of operational and installation costs of new telecommunications networks, since an existing infrastructure is used as a means of data transmission. Such advantage provided by the PLC technology becomes a factor of relevance in a project when considering the installation of access networks to telecommunications systems, sector which is responsible for around $50 \%$ of all infrastructure investment in the area [1].

PLC technology may be classified into two main categories: Broadband PLC (BB-PLC) and Narrowband PLC (NBPLC) [2]. The BB-PLC category was initially designed aiming household applications [3], however, documents such as the IEEE 1901-2010 standard [4] and the ITU-T G.9960-2010 recommendation [5] have regulated its use also for applications in public power grids, given its promising characteristics for smart meters and advanced networks control applications, as addressed by [6].

Despite having attractive advantages from the economic and technological point of view, PLC systems may present degradation of their operation for such types of applications, due to electromagnetic interference between devices connected to the power grid [7].

Considering the PLC technology, its operational characteristics and the interest in its applicability to telecommunication systems together with electric power grids, in this article is performed an assessment of the performance of a data transmission system using BB-PLC devices. For this, it is 
considered situations where the BB-PLC system is subject to adverse conditions of electromagnetic interference in a conducted and radiated form, to which it would be exposed under normal conditions of use.

The followings chapters of this article present a brief approach about some existing regulations for BB-PLC systems, in section II, followed by an introduction about the Orthogonal Frequency Division Multiplexing (OFDM) modulation technique, in section III, the tests methodology and description, in section IV, the results and discussions about this study, in section V and, finally, the conclusions are presented in section VI.

\section{BB-PLC REGULATION}

In order to standardize the technology, researchers and institutions worldwide have been working together in the development of rules and standards that technology must respect, according to the different regions of use, where disturbance limits are established to ensure the electromagnetic compatibility of such devices.

The IEEE 1901-2010 International Standard addresses on broadband devices operating in power networks with a transmission frequency below $100 \mathrm{MHz}$, focusing on the balanced and efficient use of the communication channel of the power network by all broadband devices classes that use this channel [4].

The ITU-T G.9960-2010 Recommendation specifies system architecture and functionality for all physical layer components of home network transceivers designed for data transmission, including transmission over power grids [5].

In Europe, the EN 55022 Standard applies to the regulation of information technology equipment by specifying limits for spurious signals generated in the frequency range of 9 $\mathrm{kHz}$ to $400 \mathrm{GHz}$ for class $\mathrm{A}$ and B equipment [8], applying therefore to the certification of electromagnetic compatibility in PLCs.

In United States (US), the FCC part 15 Regulation contains technical specifications for several telecommunications equipment, including devices designed to be used in connection with public power grids [9], such as PLCs.

In Brazil, in order to regulate the technology of data transmission through power grids, the National Telecommunications Agency (ANEEL) published in 2009, the Normative Resolution $\mathrm{n}^{\circ}$ 527, which approves the regulation on use conditions of radio frequencies for broadband systems through electric power grids. [10].

The Normative Resolution $\mathrm{n}^{\circ}$ 527establishes the technical characteristics necessary for the proper functioning of the broadband device in its specified frequency range, and determines limits of radiation caused by broadband communication systems through electric power grids (BPL) operating at low and medium voltage, as well as exclusion bands, in which such devices may not cause unwanted radiation, since they are frequency bands allocated to the Aeronautical Mobile and Amateur Mobile Service.

The ANEEL also provides requirements and test procedures applicable to the BPL equipment certification in Brazil, classified as Category II [11].

The PLCs devices used in this work present a compliance declaration with the specifications for a Class B digital device, according to FCC Part 15, the US regulation cited.

\section{OFDM MODULATION}

The performance of the data transmission system through power grids is significantly affected by phenomena such as noise, signal attenuation and multipath propagation, characteristics common to power network channels, since they are not specifically designed for data transmission purposes [12], [13].

In some channels of power distribution network, the number of grid interconnections between transmitter and receiver and their load and branch characteristics cause transmission and reflection of signals among transmission line segments. These phenomena lead to the degradation of propagated information [12]. To mitigate the degradations of the transmitted signal, several techniques are employed; one of them is the type of modulation applied to the signal.

The modulation technique widely used in the PLC technology is the OFDM. In this signal modulation method, a highspeed data stream is divided into a parallel low rate data stream, where these information sets are modulated in QPSK (Quadrature Phase Shift Keying) or QAM (Quadrature Amplitude Modulation) using different orthogonal subcarriers in a frequency range [12]. The modulation technique coding, then provides bits in redundancy, in such a way as multiple signals are sent at different frequencies, allowing the receiver to retrieve bits lost due to impulsive noise [14].

The OFDM modulation enables persistent narrow band interference to be easily manipulated by disconnecting their respective subcarriers [15]. In this way, the OFDM modulation technique is able to handle the phenomena of multipath propagation more effectively than single-subcarrier methods.

\section{METHODOLOGY AND DESCRIPTION OF TESTS}

The tests to evaluate the performance of the BB-PLC system, when it is subject to electromagnetic interference caused by lighting devices, were performed at the Technical Lighting Laboratory of the Federal University Fluminense (LABLUXUFF), which is active in the lighting product certification area.

All measurements were performed in a shielded room with attenuation of radio frequencies up to $30 \mathrm{MHz}$ and dimensions of $3.4 \mathrm{~m} \mathrm{x} 5.3 \mathrm{~m}$.

The BB-PLC devices used are specified with OFDM modulation technique and applied for low voltage usage. As interference sources for the tests, lighting devices were used, since such equipments work with power converters operating in frequency bands that may overlap the BB-PLC operating band.

For measuring the frequency spectrum of the lamps used as interference source and the BB-PLC system, the following equipments were used:

- Line Impedance Stabilization Network (LISN) - 9kHz$30 \mathrm{MHz}$ - CISPR 16 specification [16];

- Spectrum Analyzer;

- 13 bands Pre-Selector;

- $2.5 \mathrm{~mm}^{2}$ copper power cables.

In the interference tests and measurements of data transmission capacity, two PLCs with the same specifications and manufacturer were used, each one connected to a notebook with GNU/Linux operational system, one operating as a client and one as a server. The LISN network was used to power the PLCs, in order to provide controlled impedance and decouple the test circuit from the supply mains.

For measurements of the maximum transmission rate achievable by the system, under the analyzed conditions, it was used the software tool iperf3, which allows the injection of data packets for the performance evaluation of data transmission networks. 
Measurements of electric and magnetic field strength of samples used as sources of radiated electromagnetic interference were also performed, such as their influence on the system data transmission capacity. For these measurements, it was used a broadband non-ionizing radiation (NIR) meter, designed for measurements in the frequency range of $3 \mathrm{kHz}$ to $60 \mathrm{GHz}$.

As non-controlled electromagnetic interference source, four Compact Fluorescent Lamps (CFL) and three LED Lamps with different power and operating voltage were used, as shown in Table 1.

Table 1: Lamps used as Electromagnetic Interference Source.

\begin{tabular}{|l|l|l|l|}
\hline Source Code & \multicolumn{1}{|c|}{ Technology } & \multicolumn{1}{|c|}{$\begin{array}{c}\text { Power } \\
(\mathrm{W})\end{array}$} & \multicolumn{1}{|c|}{$\begin{array}{c}\text { Voltage } \\
(\mathrm{V})\end{array}$} \\
\hline AM01 & LED & 20 & Bivolt \\
\hline AM02 & LED & 9 & Bivolt \\
\hline AM03 & LED & 6.5 & Bivolt \\
\hline AM04 & CFL & 45 & 127 \\
\hline AM05 & CFL & 45 & 220 \\
\hline AM06 & CFL & 20 & 127 \\
\hline AM07 & CFL & 20 & 220 \\
\hline 6 Samples Set & LED and CFL & 155.5 & 127 \\
\hline 6 Samples Set & LED and CFL & 155.5 & 220 \\
\hline
\end{tabular}

Source: Authors, (2020).

Of these selected interference sources, two are LED lamps complying with the Brazilian electromagnetic compatibility specifications, according to CISPR 15 [17], one is a noisy spectrum LED lamp that exceeds the limits of electromagnetic disturbances acceptable by Brazilian regulations and the last four are noisy spectrum CFLs. The noisy spectrum samples were selected in order to know the performance of the BB-PLC system under such adverse interference conditions.

The electromagnetic compatibility tests for CFLs are not mandatory in the Brazilian market, however, for this study, measurements were performed on the CFLs samples used as interference sources and compared to the limits of the CISPR 15 standard. As result of such tests, the CFLs presented a noisy characteristic spectrum, thus not meeting the standard specifications.

The less noisy lamps, in accordance with CISPR 15 standard, are the samples coded as AM02 and AM03. The LED lamp coded as AM01 and all used CFLs, are samples with noisy characteristic spectrum in the frequency range of $9 \mathrm{kHz}$ to $30 \mathrm{MHz}$, thus not complying with the limits established by such standard.

The last two interference sources presented in Table 1 as "Set of 6 Samples", comprise two groups of 6 lamps, containing CFLs and LEDs, the first group operating at the voltage of $127 \mathrm{~V}$ and the second group at the voltage of $220 \mathrm{~V}$. Such interference sources were inserted in parallel in the PLCs power circuit to analyze the effects of the electromagnetic interference caused by the set on the system data transmission.

As mentioned before, LED lamps and CFLs, have typical operation frequency range overlapping the PLCs band. Since they emit conducted electromagnetic interference in the band of $9 \mathrm{kHz}$ to $30 \mathrm{MHz}$ (band with specified interference limits [17], and the used PLCs operate at the frequency range of $2 \mathrm{MHz}$ to $30 \mathrm{MHz}$.

Figure 1 shows the frequency spectrum of the BB-PLC system used and the Quasi-Peak (QP) and Average (AV) limits specified by the CISPR 15 standard [17] for lighting devices, to which only AM02 and AM03 interference sources comply. The peak values of three samples frequency spectrum are also presented in Figure 1, which are: the sample peak value with the highest noise level among those used (AM01-blue line) and the peak values of the models samples most commonly used in homes, where, one is of LED technology (AM02-purple line) and another is of CFL technology (AM06-green line).

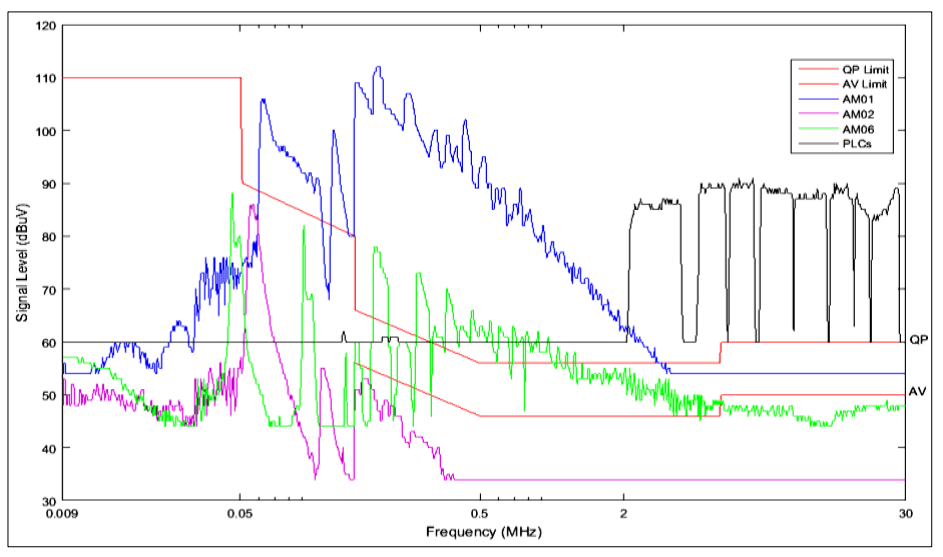

Figure 1: Characteristic frequency spectrum of the lamps used as interference source, PLCs and limits specified by the CISPR 15 standard for conducted electromagnetic disturbances of lighting devices.

Source: Authors, (2020).

The data rate measurements for performance analysis of the BB-PLC system, when subject to electromagnetic interference caused by lighting technologies, were performed considering two different forms of interference, the form conducted by the mains terminals and radiated in order to generate electric and magnetic field, as discussed in the sub-items below.

\section{IV.1 MAINS TERMINALS ELECTROMAGNETIC INTERFERENCE}

For measurement of the BB-PLC system performance, when subject to conducted electromagnetic interference, three different scenarios were considered, where the length of power cables between PLCs and interference source and the connection manner were modified. In both measurement scenarios the LISN network powering the used devices and the same samples as sources of electromagnetic interference were used. The connection of the PLCs and interference sources was made in the same circuit, in parallel.

The test settings were designed considering IEC CISPR 16-2-1 standard specifications [18], which determine measurement methods for conducted electromagnetic interference. Therefore, all measurements were performed inside a shielded room, on a table of non-conductive material and $80 \mathrm{~cm}$ high. The minimum distance of the equipment under test (PLCs) for the reference ground plane (metallic wall) was $40 \mathrm{~cm}$ and for the LISN network was $80 \mathrm{~cm}$, according to [18].

Once the used PLC devices are specified as bivolt and may operate at two voltage levels $(127 \mathrm{~V}$ and $220 \mathrm{~V})$, the measurements of the system data transmission rate, when influenced by conducted electromagnetic interference, were performed for both system operating voltages.

It was established a data transmission time of 120 seconds between the two PLCs and, for each of the interference sources described in Table 1, 10 measurements of the data rates were performed for subsequent determination of the mean value among them, in order to obtain a better confidence level in results.

Figure 2 shows the schematic with the scenario 1 test setup. In this scenario, it was considered $80 \mathrm{~cm}$ for the cable length between the PLCs and the connection point of the interference source. It was also used an $80 \mathrm{~cm}$ length cable to energize the 
interference source, in order to mitigate possible influences of radiated disturbances in data transmission, since this scenario aims to analyze the influence of conducted interferences. Such configuration of the interference source power cable was also maintained for scenario 2 .

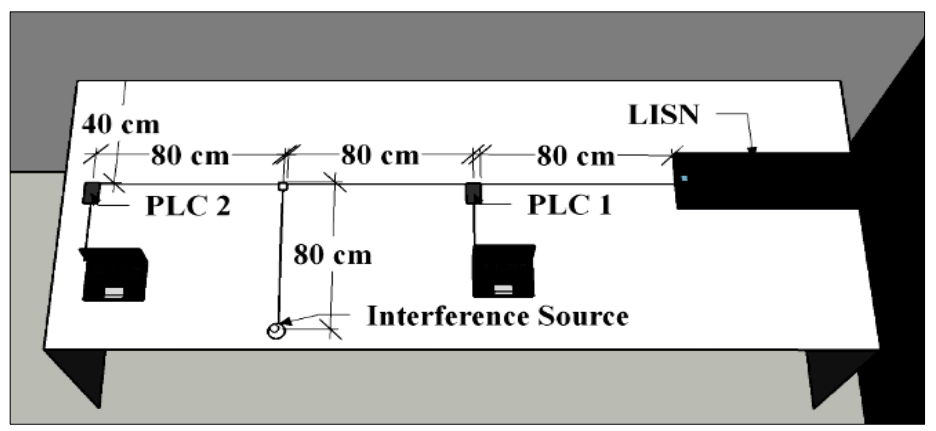

Figure 2: Measurement of the influence of conducted electromagnetic interference on the BB-PLC system performance - Scenario 1.

Source: Authors, (2020).

The second scenario adopted for analysis of the BB-PLC system performance is represented by the scheme of Figure 3. In this scenario, the length of the power cables between the PLCs and the connection point of the interference source was reduced to 40 $\mathrm{cm}$, aiming to know the cable influence on the system data transmission rate.

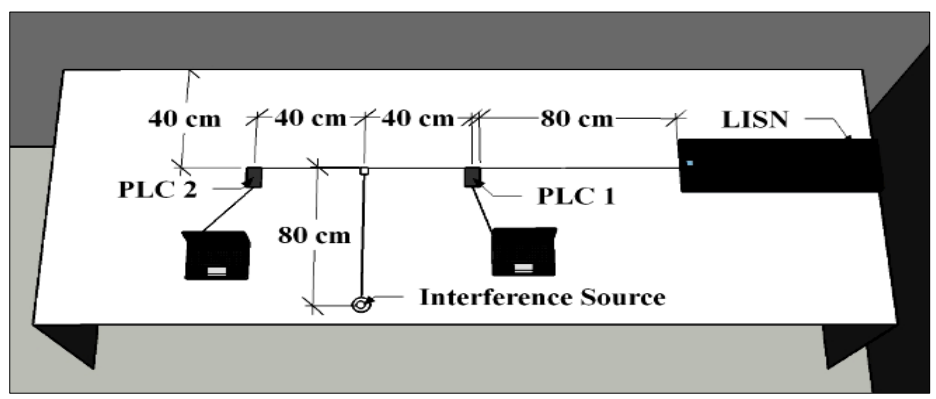

Figure 3: Measurement of the influence of conducted electromagnetic interference on the BB-PLC system performance - Scenario 2.

Source: Authors, (2020).

The scenario 3, represented by the scheme of Figure 4, comprises the measurements performed for the system where the PLCs and the interference source are connected directly to the LISN by $80 \mathrm{~cm}$ length cables. The devices were arranged in order to keep a distance of $40 \mathrm{~cm}$ from each other.

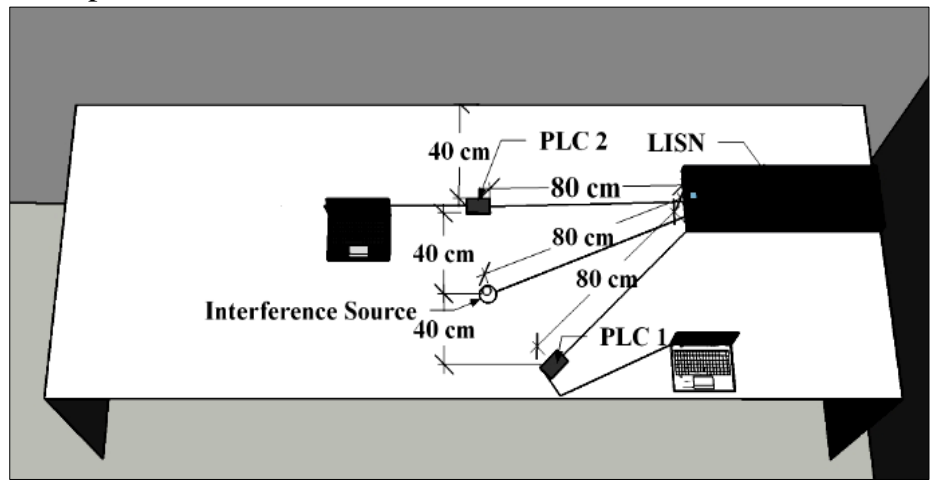

Figure 4: Measurement of the influence of conducted electromagnetic interference on the BB-PLC system performance - Scenario.

Source: Authors, (2020).

\section{IV.2 RADIATED ELECTROMAGNETIC INTERFERENCE}

In order to know also the influence of the radiated electromagnetic interference in the data transmission through power grids, using a BB-PLC system, measurements of the data transmission rate when influenced by electric $(\mathrm{E})$ and magnetic $(\mathrm{H})$ field generated by LED lamps and CFLs, were performed. In the tests the PLCs devices were connected in a different circuit from the interference source, in order to eliminate the influence of the electromagnetic interference conducted by the mains terminals. In this way, the PLCs were energized through the LISN, while the interference source was connected to a second supply circuit.

As non-controlled interference source, the lamps coded as AM01, AM02, AM05 and AM07 in Table1I were used.

Initially, measurements of the electric and magnetic field strength generated by the interference sources were performed, using a NIR meter. The measuring equipment was positioned 20 $\mathrm{cm}$ distant from the interference source and, the measurement time was established in 6 minutes, considering, for the final analyses, the mean value of the measurements performed in this time interval. The measured values of the field strength generated by the used samples and by the PLCs are presented in Table 2.

Table 2: Electric and Magnetic Field Strenght generated by devices used for Radiated Electromagnetic Interference Tests.

\begin{tabular}{|c|c|c|}
\hline Device & $\mathbf{E ~ ( V / m ) ~}$ & $\mathbf{H}(\mathbf{A} / \mathbf{m})$ \\
\hline PLCs & 0.18 & 0.0075 \\
\hline AM01 & 0.39 & 0.0079 \\
\hline AM02 & 0.2 & 0.0084 \\
\hline AM05 & 20.17 & 0.0086 \\
\hline AM07 & 6.78 & 0.0088 \\
\hline \multicolumn{2}{|c|}{ Source: Authors, (2020). }
\end{tabular}

It is possible to note that the CFLs (AM05 and AM07) are the ones with higher electric field strength. Based on a study already performed in [19], such behavior was expected for this lighting technology.

For measurements of data transmission rate under the influence of radiated electromagnetic interference, transmission time of 3 minutes was established. The measurements were performed considering two scenarios, where the distance between the interference source and the PLCs was modified. In the first scenario, a distance of $1 \mathrm{~m}$ was considered between the interference source and each of the PLCs, as shown in Figure 5; in the second scenario, this distance was reduced to $50 \mathrm{~cm}$, as presented in Figure 6.

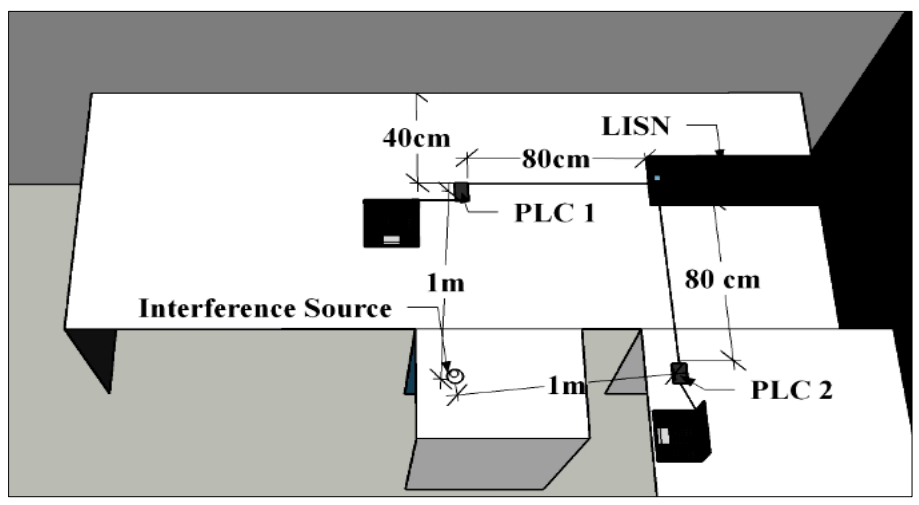

Figure 5: Measurement of the influence of radiated electromagnetic interference on the BB-PLC system performance - Scenario 1.

Source: Authors, (2020). 


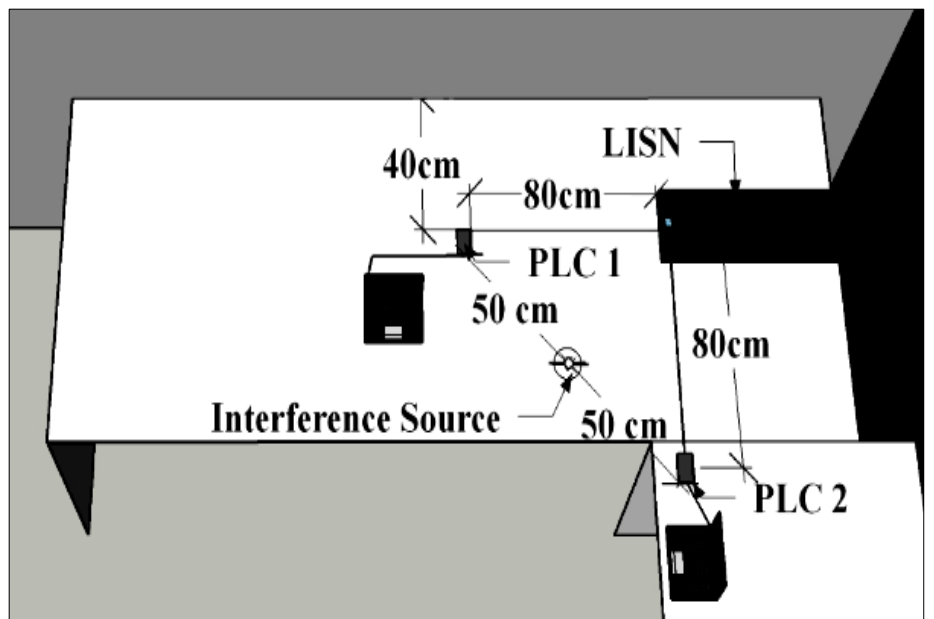

Figure 6: Measurement of the influence of radiated

electromagnetic interference on the BB-PLC system performance - Scenario 2.

Source: Authors, (2020).

\section{RESULTS AND DISCUSSIONS}

In this section, results and discussions are presented, in order to analyze the performance of the BB-PLC system for each of the interference forms and scenarios discussed in this study.

\section{V.1 BB-PLC SYSTEM PERFORMANCE WHEN SUBJECT TO CONDUCTED ELECTROMAGNETIC INTERFERENCE CAUSED BY LIGHTING TECHNOLOGIES}

Figures 7 and 8 present the results obtained for measurements of data transmission rate, when the BB-PLC system is subject to mains terminal electromagnetic interference caused by lighting devices

Figure 7 shows the results for the system operating at the voltage of $127 \mathrm{~V}$, with the bivolt samples and those operating only at 127V (AM04 and AM06). Figure 8 shows the results for the system operating at the voltage of $220 \mathrm{~V}$, with the bivolt samples and those operating only at $220 \mathrm{~V}$ (AM05 and AM07).

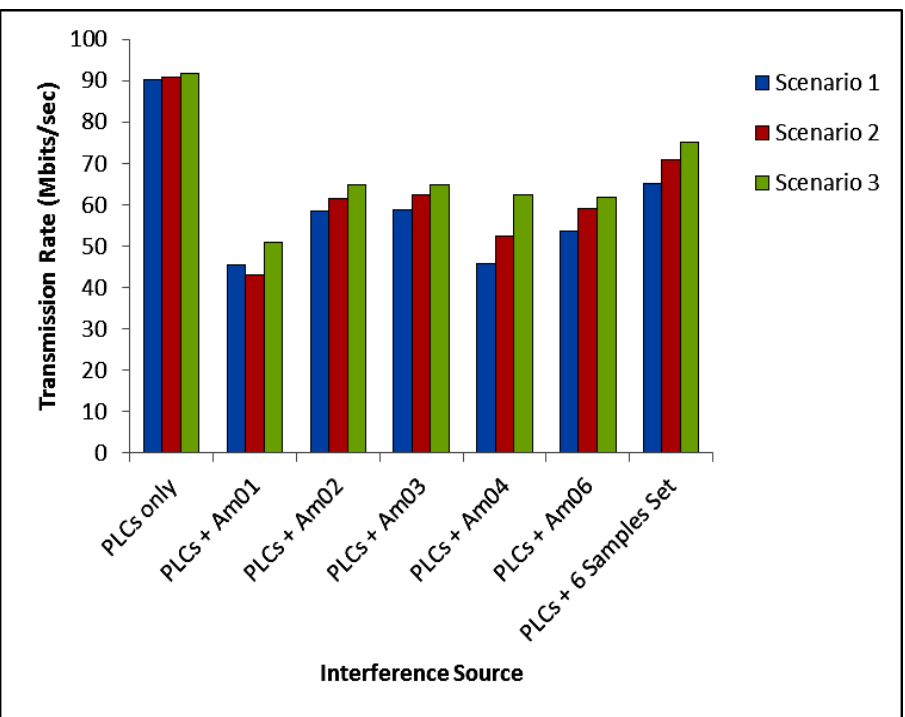

Figure 7: Transmission rate of the BB-PLC system operating at $127 \mathrm{~V}$ with insertion of conducted electromagnetic interference. Source: Authors, (2020).



Figure 8: Transmission rate of the BB-PLC system operating at $220 \mathrm{~V}$ with insertion of conducted electromagnetic interference. Source: Authors, (2020).

The columns identified in the figures as "PLCs only" refer to the BB-PLC system transmitting data without insertion of interference source into its power circuit. The other columns sets in the figures refer to the system transmitting data with the individual insertion of each of the interference sources shown in Table 1.

It is possible to observe by analyzing the graphs that the data transmission rate variation among the different scenarios is small for both analyzed voltage levels. The largest results difference among scenarios was found for insertion of the interference source AM04, in the $127 \mathrm{~V}$ voltage level, between scenarios 1 and 3, corresponding to $18 \%$, calculated relative to the maximum transmission rate value, measured for the BB-PLC system transmitting data without the insertion of an interference source. The other variations of the transmission rates among the scenarios were lower than $11 \%$, for both voltage levels.

The interference source that most affected the data transmission rate of the BB-PLC system was the AM01 source, for the system operating at $127 \mathrm{~V}$, in the second scenario. Such interference source caused a transmission rate reduction of $53 \%$, due to conducted electromagnetic interference.

\section{V.2 BB-PLC SYSTEM PERFORMANCE WHEN SUBJECT TO RADIATED ELECTROMAGNETIC INTERFERENCE CAUSED BY LIGHTING TECHNOLOGIES}

Figure 9 shows the results of the data transmission rate obtained for the BB-PLC system, when operating under influence of radiated electromagnetic interference caused by LED and CFL lamps technologies.

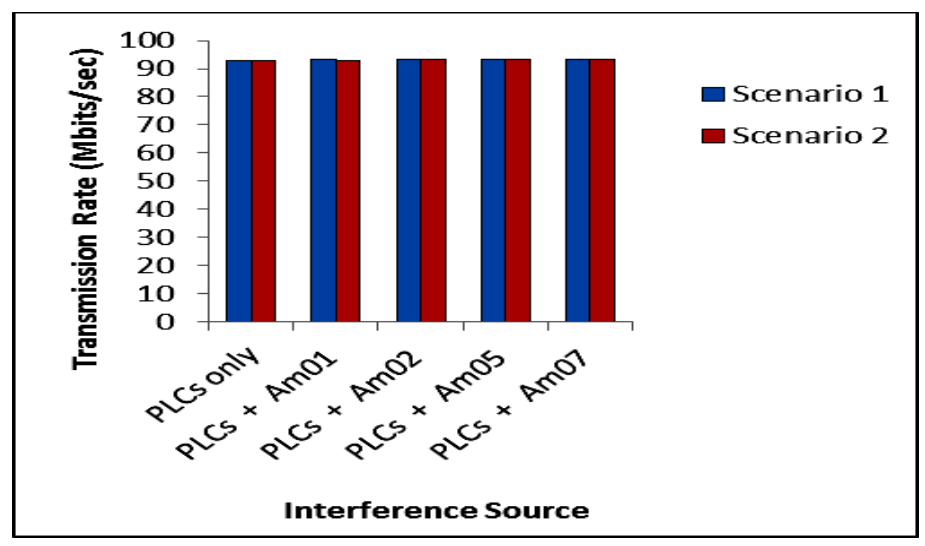

Figure 9: Transmission rate of the BB-PLC system operating with insertion of radiated electromagnetic interference.

Source: Authors, (2020). 
It is possible to observe by analyzing the graph presented in Figure 9 that the data transmission rate variation among the analyzed scenarios of radiated electromagnetic interference conditions, when existing, is small, occurring only in two occasions: insertion of the interference source AM01 and the interference source AM05; where, $0.43 \%$ and $0.11 \%$ are the variation values for each occasion, respectively, calculated relative to the maximum transmission rate value that was measured for the system operating without insertion of interference source.

It is possible to note also that the radiated electromagnetic interference, caused by the used lighting devices, does not interfere significantly in data transmission of the BB-PLC system, which maintained its transmission rate around $90 \mathrm{Mbits} / \mathrm{sec}$, even when approximating the radiated interference sources in scenario 2 .

\section{CONCLUSIONS}

This work presented a series of tests performed in order to evaluate the BB-PLC system performance, when it is subject to adverse conditions of electromagnetic interference present in the data transmission channels in power distribution networks.

Electromagnetic interference conducted by the mains terminal and radiated electromagnetic interference were considered in this study. As interference sources, CFLs and LED lamps were used, once these devices emit noise in a frequency range that overlaps the transmission band of BB-PLC systems, besides being equipment with possibility of operation in the same circuit and environment of such system.

In the analyzed data transmission system, two PLCs devices of the same manufacturer and specifications were used. Such PLCs use OFDM signal modulation technology and are designed for use in low voltage

The analyzed BB-PLC system presented good performance when subject to radiated electromagnetic interference caused by the used lamps technologies; however, it showed considerable sensitivity to conducted electromagnetic interferences caused by such devices in the mains terminals, since, a reduction of up to $53 \%$ in data transmission rate could be found for insertion of this interference form.

By analyzing the different scenarios discussed, it is also possible to conclude that the BB-PLC system suffers small variation with the length of the power cable between the two PLCs devices, and presented a low oscillation in the data transmission rate among the scenarios discussed.

As mentioned in item I of this work, data transmission technology through power grids is promising for both telecommunication and electric power systems, due to its applicability, among others, to smart grids. Therefore, it is pertinent to dedicate attention to topics involving existing limitations for such technology, in order to mitigate obstacles to its advancement.

Limitations of the BB-PLC system involving electromagnetic interference may be moderated by measures to be adopted in the transmission channels, the power grids, through filter application, for example; or by adopting improvements in the PLC technology, which may be done through the implementation of bit allocation algorithms aiming the maximization of data transmission rate, considering transmission channels with noisy characteristic spectrum and low signal-to-noise ratio (SNR).

Algorithms that provide adaptive modulation according to the transmission channel conditions, in order to maximize the reachable margin and/or data rate, have been proposed by several researchers in the last decades [20]-[31], and may represent a promising technique to implement improvements in data transmission technology through power distribution networks.

\section{REFERENCES}

[1] H. Hrasnica, A. Haidine, and R. Lehnert. Broadband powerline communications networks: network design, 2004, Chichester, England: John Wiley \& Sons Ltd..

[2] A. Usman and S. H. Shami. Evolution of Communication Technologies for Smart Grid applications, Renew. Sustain. Energy Rev., 19, p.p. 191-199, 2013.

[3] N. Graf, I. Tsokalo, and R. Lehnert. Validating broadband PLC for smart grid applications with field trials, in 2017 IEEE International Conference on Smart Grid Communications (SmartGridComm), Dresden, Germany, p.p. 497-502, 2017.

[4] Institute of Electrical and Electronics Engineers and IEEE-SA Standards Board. IEEE standard for broadband over power line networks: medium access control and physical layer specifications, 2010, New York: Institute of Electrical and Electronics Engineers.

[5] Telecomunication Standardization Sector of ITU. Unified highspeed wire-line based home networking transceivers - System architecture and physical layer specification, 2010, International Telecommunication Union.

[6] A. Mengi, S. Ponzelar, and M. Koch. The ITU-T G.9960 broadband PLC communication concept for smartgrid applications, in 2017 IEEE International Conference on Smart Grid Communications (SmartGridComm), Dresden, Germany, p.p. 492-496, 2017.

[7] C. R. Paul. Introduction to electromagnetic compatibility, 2006, 2nd ed. Hoboken, N.J: Wiley-Interscience.

[8] European Committee for Electrotechnical Standardization. Information technology equipment - Radio disturbance characteristics - Limits and methods of measurement, 2010, CENELEC.

[9] Federal Communications Commission - FCC. FCC Part 15 Radio Frequency Devices, FCC.

[10] Agência Nacional de Telecomunicações - Anatel. Resolução no 527, de 8 de abril de 2009, 2009, Anatel.

[11] Agência Nacional de Telecomunicações - Anatel. Requisitos técnicos e procedimentos de ensaios aplicáveis à certificação de produtos para telecomunicações de categoria II, 2018, Anatel.

[12] M. Muhaimin and S. P. Majumder. Performance analysis of an OFDM broadband power line communication (BPLC) system using multipath channel models, in 2014 International Conference on Electrical Engineering and Information \& Communication Technology, Dhaka, Bangladesh, p,p. 1-5, 2014.

[13] M. M. Rahman and S. P. Majumder. Performance improvement of a power line communication system using OFDM under the effect of fading and impulsive noise with diversity reception, in 7th International Conference on Broadband Communications and Biomedical Applications, Melbourne, Australia, p.p. 30-34, 2011.

[14] Electricity metering - Data exchange over powerline - Part 2: Lower layer profile using OFDM modulation type 2. Available online:

http://www.erdf.fr/sites/default/files/documentation/G3_Specifica tions_\%20low_\%20layers.pdf (Accessed November 22, 2018).

[15] N. Hadaschick. Techniques for UWB-OFDM, Institute for Communication Technologies and Embedded Systems (ICE). Available online: https://www.ice.rwth- 
aachen.de/research/algorithms-projects/entry/detail/techniquesfor-uwb-ofdm/ (Accessed November 22, 2018).

[16] International Electrotechnical Commission (IEC). Specification for radio disturbance and immunity measuring apparatus and methods - CISPR 16 Part 1-2: Radio disturbance and immunity measuring apparatus - Coupling devices for conducted disturbance measurements, 2014, IEC CISPR.

[17] Associação Brasileira de Normas Técnicas - ABNT. ABNT NBR IEC CISPR 15 - Limites e métodos de medição das radioperturbações características dos equipamentos elétricos de iluminação e similares, 2014, Associação Brasileira de Normas Técnicas.

[18] International Electrotechnical Commission (IEC). Specification for radio disturbance and immunity measuring apparatus and methods - CISPR 16 Part 2-1: Methods of measurement of disturbances and immunity - Conducted disturbance measurements, 2014, IEC.

[19] S. Aerts et al.. Exposure to electric and magnetic fields at intermediate frequencies of household appliances. in 2017 International Applied Computational Electromagnetics Society Symposium - Italy (ACES), Florence, p.p. 1-2, 2017.

[20] P. S. Chow, J. M. Cioffi, and J. A. C. Bingham. A practical discrete multitone transceiver loading algorithm for data transmission over spectrally shaped channels, IEEE Trans. Commun., vol. 43, no. 2/3/4, p.p. 773-775, 1995.

[21] A. Leke and J. M. Cioffi. A maximum rate loading algorithm for discrete multitone modulation systems," in GLOBECOM 97, in IEEE Global Telecommunications Conference. Conference Record, Phoenix, AZ, USA, vol. 3, p.p. 1514-1518, 1997.

[22] E. Bedeer, O. A. Dobre, M. H. Ahmed, and K. E. Baddour. Optimal bit and power loading for OFDM systems with average BER and total power constraints, in 2012 IEEE Global Communications Conference (GLOBECOM), Anaheim, CA, USA, p.p. 3685-3689, 2012.

[23] E. Guerrini, G. Dell'Amico, P. Bisaglia, and L. Guerrieri. Bitloading algorithms and SNR estimate for HomePlug AV, in 2007 IEEE International Symposium on Power Line Communications and Its Applications, Pisa, Italy, pp. 77-82, 2007.

[24] E. Guerrini, L. Guerrieri, D. Veronesi, P. Bisaglia, and R. Cappelletti. LLR-based Bit-loading Algorithm and its Applications to HomePlug AV over OPERA Power-line Channels with Impulsive Noise, J. Commun., vol. 4, no. 7, 2009.

[25] K. S. Al-Mawali, A. Z. Sadik, and Z. M. Hussain.Simple discrete bit-loading for OFDM systems in power line communications, in 2011 IEEE International Symposium on Power Line Communications and Its Applications, Udine, Italy, p.p. $267-$ 270, 2011.

[26] Y. George and O. Amrani. Bit loading algorithms for OFDM," in International Symposium onInformation Theory - ISIT 2004. Proceedings., Chicago, Illinois, USA, 2004, p.p. 391-391.

[27] D. Daly, C. Heneghan, and A. D. Fagan. Power- and bitloading algorithms for multitone systems, in 3rd International Symposium on Image and Signal Processing and Analysis. ISPA 2003. Proceedings of the, Rome, Italy, 2003, vol. 2, p.p. 639-644.

[28] H. Rohling and C. Fellenberg. Successive bit loading scheme, Electron. Lett., vol. 45, no. 4, p. 214, 2009.
[29] L. Yang, M. Yu, L. Zhou, and Y. Xu. Adaptive bit loading algorithm of shortwave broadband OFDM system, in 2011 Second International Conference on Mechanic Automation and Control Engineering, Inner Mongolia, China, p.p. 49-52, 2011.

[30] S. Nagaraj. Improving power efficiency of coded OFDM with feedback, IEEE Trans. Commun., vol. 57, no. 9, p.p. 2541-2544, 2009.

[31] Y.Li and G. Su, An adaptive modulation and power-allocation algorithm in OFDM system, in 2004 International Conference on Communications, Circuits and Systems (IEEE Cat. No.04EX914), Chengdu, p.p. 339-343 Vol.1, 2004. 